\title{
Combinaison thérapeutique par cellules $T$ à récepteurs antigéniques chimériques anti-CD19 et anti-antigène de maturation des cellules B du myélome multiple en rechute ou réfractaire
}

Nicolas Stocker

es progrès thérapeutiques récents, tels que le développement des agents immunomodulateurs, des inhibiteurs du protéasome ou des anticorps monoclonaux, ont significativement amélioré la survie des patients atteints de myélome multiple [1]. Tandis que les rechutes restent fréquentes, les lymphocytes $\mathrm{T}$ à récepteur antigénique chimérique (CAR-T) ciblant l'antigène de maturation des lymphocytes B (BCMA) représentent aujourd'hui une alternative thérapeutique incontournable [2]. Cependant, des études ont récemment mis en évidence des contingents plasmocytaires faiblement différenciés et exprimant la cible $\mathrm{CD}_{19^{+}}$[3], suggérant la perspective d'un traitement combiné ciblant BCMA et CD19. Cet essai de phase 2 a évalué une combinaison de CAR-T anti-CD19 et anti-BCMA chez des patients atteints de myélome multiple en rechute ou réfractaire (MMRR) [4].

Entre mai 2017 et janvier 2019, vingt-deux patients atteints de MMRR ont été inclus dans cette étude. Vingt et un patients reçurent des CAR-T autologues anti-CD19 et anti-BCMA, un patient ne put les recevoir en raison de la progression rapide $\mathrm{du}$ MMRR. Les patients recevaient un conditionnement lymphodéplétant par fludarabine $\left(30 \mathrm{mg} / \mathrm{m}^{2} / \mathrm{j}\right.$ pendant trois jours) et cyclophosphamide $\left(750 \mathrm{mg} / \mathrm{m}^{2}\right)$, avant l'administration de CAR-T anti-CD19 humanisés $\left(1 \times 10^{6} / \mathrm{kg}\right)$ et de CAR-T anti-BCMA murins $\left(1 \times 10^{6} / \mathrm{kg}\right)$. L'âge médian des patients était de 58 ans (50-61) et le délai médian depuis le diagnostic de 24 mois (8-120). Les patients avaient reçu une médiane de six lignes antérieures de traitement (4-17).

Avec un suivi médian de 179 jours (IQR 72-295) après administration des CAR-T, $95 \%$ des patients $(n=20)$ étaient en réponse clinique : $43 \%(\mathrm{n}=9)$ en réponse complète stringente, $14 \%(n=3)$ en réponse complète, $24 \%(n=5)$ en réponse partielle de très bonne qualité et $14 \%(n=3)$ en réponse partielle. Parmi les vingt patients répondeurs, $85 \%(\mathrm{n}=17)$ n'ont pas progressé pendant le suivi de l'étude. Des corrélations négatives étaient observées entre les pics de concentration des CAR-T anti-CD19 et antiBCMA à J7, et les taux de maladie résiduelle détectable (respectivement $R^{2}=-0,48, \quad p=0,027$; et $\left.\mathrm{R}^{2}=-0,52, \mathrm{p}=0,016\right)$. En revanche, si le pic de concentration à J7 du CAR-T anti-BCMA était associé au nombre de plasmocytes médullaires $\left(\mathrm{R}^{2}=0,62, \mathrm{p}=0,030\right)$; aucune association n'était observée avec le CAR-T anti-CD19.

Les principaux effets indésirables étaient des syndromes de relargage cytokinique et des toxicités hématologiques. $90 \%$ des patients $(\mathrm{n}=19)$ ont ainsi présenté un syndrome de relargage cytokinique : $\quad 86 \%$ ( $\mathrm{n}=18$ ) de grade $1-2$, et un unique (5\%) de grade 3 . Chez ces patients, le délai médian d'apparition de la fièvre était notamment de neuf jours (1-15). Sur le plan des toxicités hématologiques, $95 \%$ des patients $(\mathrm{n}=20)$ ont présenté une toxicité de grade $\geq 3: 86 \%(n=18)$ de leucopénie, $62 \%$ (n = 13) d'anémie et $62 \%(n=13)$ de thrombopénie. Enfin, si la lymphopénie B était systématiquement observée dans les soixante jours suivant l'administration des CAR-T, $80 \%$ des patients possédaient des taux normalisés de lymphocytes B à six mois post-CAR-T. En conclusion, cette étude de phase II démontre la faisabilité et l'efficacité prometteuse d'un traitement combiné par CAR-T anti-CD19 et antiBCMA chez les patients atteints d'un MMRR. En effet, cette combinaison thérapeutique permet l'obtention de taux impressionnants de réponse chez des patients lourdement prétraités et réfractaires. Enfin, si les effets indésirables semblaient acceptables dans ce contexte clinique, des études randomisées devront objectiver le gain réel de cette combinaison thérapeutique. ]

\section{Références}

[1] Kumar SK, Dispenzieri A, Lacy MQ, et al. Continued improvement in survival in multiple myeloma: changes in early mortality and outcomes in older patients. Leukemia $2014 ; 28$ : 1122-8.

[2] Brudno JN, Maric I, Hartman SD, et al. T Cells Genetically Modified to Express an Anti-B-Cell Maturation Antigen Chimeric Antigen Recepto Cause Remissions of Poor-Prognosis Relapsed Multiple Myeloma. J Clin Oncol 2018; 36 2267-80.

[3] Paiva B, Puig N, Cedena MT, et al. Differentiation stage of myeloma plasma cells: biological and clinical significance. Leukemia 2017; 31 382-92

[4] Yan Z, Cao J, Cheng H, et al. A combination of humanised anti-CD19 and anti-BCMA CAR T cells in patients with relapsed or refractory multiple myeloma: a single-arm, phase 2 trial. Lancet Haematol 2019; 6 : e521-e9. 